\title{
PIC-Based Iterative SDR Detector for OFDM Systems in Doubly-Selective Fading Channels
}

\author{
Shu Feng, Member, IEEE, Hlaing Minn, Senior Member, IEEE, Liang Yan, and Lu Jinhui
}

\begin{abstract}
OFDM data detection in doubly-selective fading channels requires high complexity due to intercarrier interferences (ICI). We present a low-complexity receiver consisting of a semidefinite relaxation (SDR) based detector and parallel interference cancellation (PIC). The entire band is divided into clusters of adjacent subcarriers. SDR is applied on each cluster while PIC tackles ICI from other clusters. An upper bound of ICI power is derived and used to omit far-away clusters in performing PIC. Finally, an adaptive detector based on PIC, PIC-based SDR and the snap-shot SNR in channel is proposed to achieve a better tradeoff between complexity and performance.
\end{abstract}

Index Terms-Maximum likelihood, parallel interference cancellation, semidefinite relaxation, intercarrier interference.

\section{INTRODUCTION}

$\mathbf{N}$ EXT-GENERATION wireless systems will support applications with a mobile speed as high as $350 \mathrm{~km} / \mathrm{h}$ (e.g., in IMT-Advanced systems). A high mobile speed results in a large Doppler spread or equivalently a fast time-variant channel, which in turns introduces inter-carrier interference (ICI) in orthogonal frequency division multiplexing (OFDM) systems and degrades the bit error rate (BER) performance significantly [1], [2]. In this paper, we will focus on signal detection of OFDM systems in frequency-selective fading channels with NDS $\geq 0.1$ (referred to as doubly-selective (DS) fading channels afterwards) where the normalized Doppler spread (NDS) is the Doppler spread $f_{d}$ normalized by the sub-carrier spacing.

For OFDM systems in DS channels, the key issue of signal detection is how to remedy the detrimental effect of ICI on BER performance. There exist several related works in the literature including a block-matrix based equalizer with $N$ DFTs of size $N$ (= number of subcarriers) [3], with 6 or 7 DFTs [4], high-complexity minimum mean-squared error (MMSE) technique combined with successive detection [5], a low-complexity two-stage equalizer [6], a low-complexity MMSE block linear equalizer [7] and its error floor issue [8], a time-frequency per-tone equalizer [9], ICI cancellation

Manuscript received April 4, 2009; revised August 9, 2009 and October 26, 2009; accepted November 8, 2009. The associate editor coordinating the review of this letter and approving it for publication was H. Nguyen.

This work is supported in part by the NSFC Grants (No. 60702028), the High Technology Research and Development Programme of China (No. 2007AA01Z268), the starting fund for science research of NJUST (AB41947), and science research developing fund of NJUST (XKF07023, AB96223).

S. Feng, L. Yan, and L. Jinhui are with the Department of Communication Engineering, EEOT, Nanjing University of SCI and TECH, Nanjing, China (e-mail: shufeng@mail.njust.edu.cn).

H. Minn and S. Feng are with the Department of Electrical Engineering, University of Texas at Dallas, Texas, USA (e-mail: hlaing.minn@utdallas.edu).

Digital Object Identifier 10.1109/TWC.2010.01.090462 approaches [10]-[13], and sphere decoders (SD) [14], [15] for MIMO systems which achieve the maximum likelihood (ML) performance with exponential complexity [16], [17]. Semidefinite Relaxation (SDR) methods have extensively been used in multi-user detection [18]-[20] in CDMA systems and multi-antenna data detection in MIMO systems [21]-[24] because they approach the ML detector performance only with polynomial complexity. An SDR detector of MIMO systems in real channels can achieve the diversity order of half of the number of receiving antennas [25]. Below, we will focus on the investigation of SDR-based detection of OFDM systems in DS fading channels, and propose an adaptive detector based on PIC detector and the proposed PIC-based iterative SDR detector together with the estimated snap-shot channel SNR to achieve a better tradeoff between performance and complexity.

Section II presents the system model. Section III describes the SDR detector and its low-complexity version. Section IV provides simulation results and discussions, and Section V gives the conclusions.

Notations: Bold letters with and without overline denote real and complex vectors and matrices, respectively. $(\cdot)^{H}$ denotes the conjugate transposition. $N$ is the number of total subcarriers and $L$ is the number of channel taps. $\mathbf{F}_{n}$ denotes the $n$-point unitary DFT matrix. $\Re(\cdot)$ and $\Im(\cdot)$ represent the real and imaginary parts, respectively. When accessing vectors or matrices, Matlab convention is adopted, e.g., $X(m: n)$ means $[X(m), X(m+1), \ldots, X(n)]^{T} . S_{\mathrm{QPSK}}^{N}=S_{\mathrm{QPSK}} \times$ $S_{\mathrm{QPSK}} \times \cdots \times S_{\mathrm{QPSK}}$ with the operator $\times$ representing the Cartesian product, $S_{\mathrm{BPSK}}^{2 N}=\{+1,-1\}^{2 N}$.

\section{SySTEM MODEL}

In DS fading channels, OFDM systems with cyclic prefix (CP) of at least $L-1$ samples is modeled as

$$
\mathbf{Y}(k)=\mathbf{H}_{k} \mathbf{X}(k)+\mathbf{W}(k)
$$

where $k$ denotes the OFDM symbol index, $\mathbf{Y}(k)$ and $\mathbf{X}(k)$ are the corresponding transmitted and received frequencydomain data vectors of size $N$, respectively. $\mathbf{W}(k)$ is an $N \times 1$ independent and identically-distributed (iid) additive white Gaussian noise (AWGN) complex random vector, $\mathbf{H}_{k}$ is an $N \times N$ frequency-domain channel matrix equal to

$$
\mathbf{H}_{k}=\mathbf{F}_{N} \mathbf{h}_{k} \mathbf{F}_{N}^{H}
$$

where $\mathbf{h}_{k}$ is constructed as

$$
\left(\begin{array}{ccccccccc}
h_{k}^{1,0} & h_{k}^{1,1} & \ldots & \ldots & \ldots & h_{k}^{1, L} & 0 & \ldots & 0 \\
0 & h_{k}^{2,0} & \ldots & \ldots & \cdots & h_{k}^{2, L-1} & h_{k}^{2, L} & \cdots & 0 \\
\vdots & \vdots & \ddots & \vdots & \ddots & \vdots & \vdots & \ddots & \vdots \\
h_{k}^{N, 1} & h_{k}^{N, 2} & \ldots & h_{k}^{N, L} & \ldots & 0 & 0 & \cdots & h_{k}^{N, 0}
\end{array}\right)
$$


with $h_{k}^{n, l}$ denoting the $l$ th complex path gain of the channel corresponding to the $n$th sampling point during the $k$ th OFDM symbol. Due to channel variations during an OFDM symbol, $\mathbf{H}_{k}$ in (1) is no longer a diagonal matrix in DS fading channels.

\section{PRoposed SDR Detector}

In this section, for the convenience of presentation, we suppose that QPSK modulation is adopted. The performance results for 16-QAM and 64-QAM will be presented in Section IV.

\section{A. Original SDR Detector for QPSK}

The maximum likelihood (ML) estimate of $\mathbf{X}(k), \widehat{\mathbf{X}}_{\mathrm{ML}}(k)$ is given by

$$
\widehat{\mathbf{X}}_{\mathrm{ML}}(k)=\underset{\mathbf{X}(k) \in S_{\mathrm{QPSK}}^{N}}{\arg \min }\left\|\mathbf{Y}(k)-\mathbf{H}_{k} \mathbf{X}(k)\right\|
$$

where $\|\cdot\|$ denotes the Euclidean norm. Equation (4) is rewritten in the real-valued form [18]-[25] as

$$
\widehat{\overline{\mathbf{X}}}_{\mathrm{ML}}(k)=\underset{\overline{\mathbf{X}}(k) \in S_{\mathrm{BPSK}}^{2 N}}{\arg \min }\left\|\overline{\mathbf{Y}}(k)-\overline{\mathbf{H}}_{k} \overline{\mathbf{X}}(k)\right\|^{2}
$$

where

$$
\begin{aligned}
\overline{\mathbf{Y}}(k)=\left[\begin{array}{l}
\Re(\mathbf{Y}(k)) \\
\Im(\mathbf{Y}(k))
\end{array}\right], \overline{\mathbf{H}}_{k}=\left[\begin{array}{cc}
\Re\left(\mathbf{H}_{k}\right) & -\Im\left(\mathbf{H}_{k}\right) \\
\Im\left(\mathbf{H}_{k}\right) & \Re\left(\mathbf{H}_{k}\right)
\end{array}\right], \\
\overline{\mathbf{X}}(k)=\left[\begin{array}{l}
\Re(\mathbf{X}(k)) \\
\Im(\mathbf{X}(k))
\end{array}\right], \overline{\mathbf{W}}(k)=\left[\begin{array}{c}
\Re(\mathbf{W}(k)) \\
\Im(\mathbf{W}(k))
\end{array}\right] .
\end{aligned}
$$

ML detector in (5) is optimal in the sense that it minimizes the probability of error given that all transmitted messages are a priori equally likely. However, it has been shown to have a complexity of $O\left(4^{N}\right)$ add-multiply operations (AMOs) or arithmetic operations(AOs). To avoid such a heavy computational load, eq. (5) is semidefinite relaxed as the following convex optimization problem [18]-[25]

$$
\min _{\mathbf{S}_{k}} \operatorname{Tr}\left(\mathbf{L}_{k} \mathbf{S}_{k}\right) \text { subject to } \mathbf{D}\left(\mathbf{S}_{k}\right)=\mathbf{e}_{2 N+1} \text { and } \mathbf{S}_{k} \succeq 0
$$

where $\mathbf{S}_{k} \succeq 0$ means that $\mathbf{S}_{k}$ is symmetric and positive semidefinite, $\mathbf{e}_{2 N+1}$ is the $(2 N+1) \times 1$ vector of all ones, $\mathbf{D}\left\{\mathbf{S}_{k}\right\} \triangleq\left[\mathbf{S}_{k}(1,1), \mathbf{S}_{k}(2,2), \cdots, \mathbf{S}_{k}(2 N+1,2 N+1)\right]^{T}$, and

$$
\mathbf{L}_{k} \triangleq\left[\begin{array}{cc}
\overline{\mathbf{H}}_{k}^{T} \overline{\mathbf{H}}_{k} & -\overline{\mathbf{H}}_{k}^{T} \overline{\mathbf{Y}}(k) \\
-\overline{\mathbf{Y}}^{T}(k) \overline{\mathbf{H}}_{k} & \overline{\mathbf{Y}}^{T}(k) \overline{\mathbf{Y}}(k)
\end{array}\right] .
$$

This procedure (7) yields an approximation to the ML, and its computational amount is about $O\left((2 N+1)^{3.5}\right)$ AOs [19], [20]. As $N$ increases, the complexity advantage of SDR over ML becomes obvious.

\section{B. Proposed PIC-based Iterative SDR Detector}

Unlike SDR detectors in MIMO systems where the complexity-determining factors - the numbers of transmit and receive antennas - are often less than 10, the SDR detector for OFDM with ICI has a complexity-determining factor $N$ ranging from 64 to 8192 . For example, when $N=128$, the SDR's complexity is $(2 N+1)^{3.5}$ (AOs) $>10^{8}$ (AOs) which becomes an obstacle for its real-time implementation on mobile terminals. To simplify the complexity, we suggest the total channel bandwidth be divided into $K$ clusters, each consisting of a group of adjacent subcarriers. Our basic idea is to address the ICI outside each cluster by using PIC and the ICI within each cluster by SDR. This scheme is abbreviated as SDRIC afterwards. The choice of $K$ is related to the coherent bandwidth of DS channels. A natural choice is that each cluster bandwidth is approximately equal to the channel coherent bandwidth $B_{c}$. Below, we take $K=4$ as an example to explain our approach. Then, (1) can be rewritten as

$$
\begin{gathered}
\mathbf{Y}(k)=\left(\begin{array}{c}
\mathbf{Y}^{1}(k) \\
\mathbf{Y}^{2}(k) \\
\mathbf{Y}^{3}(k) \\
\mathbf{Y}^{4}(k)
\end{array}\right)= \\
\left(\begin{array}{cccc}
\mathbf{H}_{k}^{1,1} & \mathbf{H}_{k}^{1,2} & \mathbf{H}_{k}^{1,3} & \mathbf{H}_{k}^{1,4} \\
\mathbf{H}_{k}^{2,1} & \mathbf{H}_{k}^{2,2} & \mathbf{H}_{k}^{2,3} & \mathbf{H}_{k}^{2,4} \\
\mathbf{H}_{k}^{3,1} & \mathbf{H}_{k}^{3,2} & \mathbf{H}_{k}^{3,3} & \mathbf{H}_{k}^{3,4} \\
\mathbf{H}_{k}^{4,1} & \mathbf{H}_{k}^{4,2} & \mathbf{H}_{k}^{4,3} & \mathbf{H}_{k}^{4,4}
\end{array}\right)\left(\begin{array}{l}
\mathbf{X}^{1}(k) \\
\mathbf{X}^{2}(k) \\
\mathbf{X}^{3}(k) \\
\mathbf{X}^{4}(k)
\end{array}\right)+\left(\begin{array}{c}
\mathbf{W}^{1}(k) \\
\mathbf{W}^{2}(k) \\
\mathbf{W}^{3}(k) \\
\mathbf{W}^{4}(k)
\end{array}\right)
\end{gathered}
$$

where the superscript $i$ of $\mathbf{Y}, \mathbf{X}$ and $\mathbf{W}$ denotes the cluster index, $\mathbf{H}_{k}^{m, n}=\mathbf{H}_{k}(m-1) N / K+1: m N / K,(n-1) N / K+$ $1: n N / K)$. From (9), the received signal vector of the $i$ th cluster is equal to

$$
\mathbf{Y}^{i}(k)=\mathbf{H}_{k}^{i, i} \mathbf{X}^{i}(k)+\underbrace{\sum_{n=1, n \neq i}^{K} \mathbf{H}_{k}^{i, n} \mathbf{X}^{n}(k)}_{\mathbf{I}_{\text {out }}^{i}(k)}+\mathbf{W}^{i}(k)
$$

where $\mathrm{i} \in\{1,2, \ldots, K\}$, and $\mathbf{I}_{\text {out }}^{i}(k)$ is the ICI from other clusters. We now describe the PIC-based SDR method.

\section{Algorithm: PIC-based SDR algorithm}

- Set $\mathbf{Y}_{\text {old }}^{i}(k)=\mathbf{Y}^{i}(k)$.

- Repeat

1) Using (6), transform the complex matrix form in (10) into the real matrix form

$$
\overline{\mathbf{Y}}^{i}(k)=\overline{\mathbf{H}}_{k}^{i, i} \overline{\mathbf{X}}^{i}(k)+\overline{\mathbf{I}}_{\text {out }}^{i}(k)+\overline{\mathbf{W}}^{i}(k) .
$$

2) Obtain the detected value of $\overline{\mathbf{X}}^{i}(k), \widehat{\overline{\mathbf{X}}}_{\mathrm{SDR}}^{i}(k), \forall i$, by viewing $\overline{\mathbf{I}}_{\text {out }}^{i}(k)$ as a noise component.

3) Convert the real $2 N \times 1$ vector back to the complex $N \times 1$ vector.

4) Remove the ICI of the $i$ th cluster for all $i$ by the expression

$$
\mathbf{Y}_{\text {new }}^{i}(k)=\mathbf{Y}_{\text {old }}^{i}(k)-\sum_{n=1, n \neq i}^{K} \widehat{\mathbf{H}}_{k}^{i, n} \widehat{\mathbf{X}}_{\mathrm{SDR}}^{n}(k) .
$$

5) Update $\mathbf{Y}^{i}(k)$ in (10) by the new vector $\mathbf{Y}_{\text {new }}^{i}(k)$. Until a predefined number $(I)$ of iterations are performed. The number $I$ may be designed offline based on its BER performance.

The complexity of the proposed algorithm is of the order $O\left(I K\left(N_{B}\right)^{3.5}+I(K-1)\left(N_{B}\right)^{2}\right)$ AOs (mainly AMOs) where $N_{B}=N / K$, the first term is due to SDR, and the second is due to PIC. The complexity reduction over the SDR is a factor of $(2 N+1)^{3.5} /\left(I K\left(N_{B}\right)^{3.5}+I(K-1)\left(N_{B}\right)^{2}\right)$ which is 
approximately equal to $K^{2.5} I^{-1}$ since $K N_{B}^{3.5} \gg(K-1) N_{B}^{2}$ for practical systems.

The proposed detector becomes SDR for $K=1$ whereas it degenerates into PIC for $K=N$. In other words, its performance is between that of PIC and that of SDR and decreases as $K$ increases. Our proposed algorithm is applicable to higher order QAM or PSK with regular constellations if a SDR scheme for high order modulation in [21], [23], [24] is used instead of the SDR detector for QPSK in this algorithm. Moreover, any detector such as SD can also replace SDR in our algorithm to implement ICI cancellation within cluster.

\section{ICI Analysis and Further Complexity Reduction}

Since ICI's from far-away clusters are negligible (as will be quantified in this section), we can further reduce the complexity by considering $2 K^{\prime}$ closest clusters to each $i$ th one instead of all $K-1$ clusters in (10). Then, the fourth step of the proposed algorithm can be further simplified as

$$
\mathbf{Y}_{\text {new }}^{i}(k) \approx\left\{\begin{array}{l}
\mathbf{Y}_{\text {old }}^{i}(k) \\
-\sum_{n=i-K^{\prime}}^{i+K^{\prime}} \widehat{\mathbf{H}}_{k}^{i, n} \widehat{\mathbf{X}}_{\mathrm{SDR}}^{n}(k), K^{\prime}<i \leq K_{o} \\
\mathbf{Y}_{\text {old }}^{i}(k)-\sum_{n=1}^{K^{\prime}+i} \widehat{\mathbf{H}}_{k}^{i, n} \widehat{\mathbf{X}}_{\mathrm{SDR}}^{n}(k) \\
-\sum_{n=K_{1}+i}^{K} \widehat{\mathbf{H}}_{k}^{i, n} \widehat{\mathbf{X}}_{\mathrm{SDR}}^{n}(k), 1 \leq i \leq K^{\prime} \\
\mathbf{Y}_{\text {old }}^{i}(k)-\sum_{n+i}^{K} \widehat{\mathbf{H}}_{k}^{i, n} \widehat{\mathbf{X}}_{\mathrm{SDR}}^{n}(k) \\
-\sum_{n=i-K^{\prime}}^{K, n \neq i} \widehat{\mathbf{H}}_{k}^{i, n} \widehat{\mathbf{X}}_{\mathrm{SDR}}^{n}(k), K_{o}<i \leq K
\end{array}\right.
$$

where $K_{o}=K-K^{\prime}$ and the above formula efficiently reduces the complexity in this step. We will design the $K^{\prime}$ required in the above reduced-complexity approach, based on the upper bound of the ICI power from other far clusters in the frequency direction. In our derivation of this ICI power bound, we assume $K \geq 4, K^{\prime} \geq 1,2 K^{\prime}+1 \leq K$, and $\left|f_{d} T_{u}\right| \leq 0.5$, where $T_{u}$ is the useful length of OFDM symbols. Below, we only consider the case of $K^{\prime}<i \leq K-K^{\prime}$, since for $i \leq K^{\prime}$ or $i>K-K^{\prime}$, the proof process is similar and the result is identical due to the cyclic property of ICI in the subcarrier domain in OFDM systems. Our proof consists of two stages. In the first stage, we will deduce the upper bound of ICI arising from a single frequency offset (SFO) $\Delta f$. In the second stage, the product of this upper bound and the Jakes' spectrum is integrated over the interval $\left[-f_{d}, f_{d}\right]$ to obtain the ICI upper bound due to the Doppler spread. An OFDM system with a SFO can be modeled as [26]

$$
\begin{array}{r}
Y_{\Delta f}^{i}(k, m)=\underbrace{a_{\Delta f} \cdot X^{i}(k, m) \cdot H^{i}(k, m)}_{U_{\Delta f}^{i}(k, m)}+I_{A}^{i}(k, m, \Delta f) \\
+I_{B}^{i}(k, m, \Delta f)+W^{i}(k, m)
\end{array}
$$

where superscript $i$ denotes the $i$ th cluster, $m$ is the index of subcarrier within the $i$ th cluster ranging from 1 to $N / K$, $Y_{\Delta f}^{i}(k, m)$ is the received symbol with SFO, $X^{i}(k, m)$ the transmitted data symbol, and $H^{i}(k, m)$ the frequency-domain channel gain without SFO. In (12), $U_{\Delta f}^{i}(k, m)$ is the desired signal, $I_{A}^{i}(k, m, \Delta f)$ denotes the ICI from $2 K^{\prime}$ clusters closest to the ith cluster, and $I_{B}^{i}(k, m, \Delta f)$ is the ICI from the remaining clusters. They are given as follows:

$$
a_{\Delta f}=\frac{1}{N} \frac{\sin \left(\pi \Delta f T_{u}\right)}{\sin \left(\pi \Delta f T_{u} / N\right)} \exp \left\{\frac{j \pi \Delta f T_{u}(N-1)}{N}\right\}
$$

$$
\begin{gathered}
I_{A}^{i}(k, m, \Delta f)=\frac{1}{N}\left(\sum_{l=\left(i-K^{\prime}-1\right) N_{B}+1}^{m^{\prime}-1}+\sum_{l=m^{\prime}+1}^{\left(i+K^{\prime}\right) N_{B}}\right) \\
\frac{X(k, l) H(k, l) \sin \left(\pi \Delta f T_{u}\right) \exp \left(\frac{-j \pi\left(l-m^{\prime}\right)}{N}\right)}{\sin \left\{\frac{\pi\left(l-m^{\prime}+\Delta f T_{u}\right)}{N}\right\} \exp \left\{\frac{-j \pi \Delta f T_{u}(N-1)}{N}\right\}} \\
I_{B}^{i}(k, m, \Delta f)=\frac{1}{N}\left(\sum_{l=1}^{\left(i-K^{\prime}-1\right) N_{B}}+\sum_{l=\left(i+K^{\prime}\right) N_{B}+1}^{N}\right) \\
\frac{X(k, l) H(k, l) \sin \left(\pi \Delta f T_{u}\right) \exp \left(\frac{-j \pi\left(l-m^{\prime}\right)}{N}\right)}{\sin \left\{\frac{\pi\left(l-m^{\prime}+\Delta f T_{u}\right)}{N}\right\} \exp \left\{\frac{-j \pi \Delta f T_{u}(N-1)}{N}\right\}}
\end{gathered}
$$

where $m^{\prime}=(i-1) N_{B}+m$ and $N_{B}=N / K$. Since PIC and SDR remove $I_{A}^{i}(k, m, \Delta f)$, it is natural to only estimate the ICI power due to $I_{B}^{i}(k, m, \Delta f)$. We define the normalized residual interference power

$$
\operatorname{ICI}_{B}^{i}(k, m, \Delta f)=\frac{E\left\{I_{B}^{i}(k, m, \Delta f)\left[I_{B}^{i}(k, m, \Delta f)\right]^{*}\right\}}{E\left\{U_{\triangle f}^{i}(k, m)\left[U_{\triangle f}^{i}(k, m)\right]^{*}\right\}}
$$

$$
\begin{aligned}
= & \sin ^{2}\left\{\frac{\pi \Delta f T_{u}}{N}\right\} \cdot\left\{g\left(-\Delta f, m+K^{\prime} N_{B},(i-1) N_{B}+m-1\right)\right. \\
& \left.+g\left(\Delta f, N_{B}-m+K^{\prime} N_{B}+1, N-(i-1) N_{B}-m\right)\right\}
\end{aligned}
$$

where

$$
g(x, m, n)=\sum_{l=m}^{n} \sin ^{-2}\left(\pi\left(l+x T_{u}\right) / N\right) .
$$

Now, we derive the upper bound of the function $g(x, m, n)$. Rewriting $g(x, m, n)$ as

$$
\begin{array}{r}
g(x, m, n)=\sum_{l=m}^{n} \frac{1}{\sin ^{2}\left(\pi\left(l+x T_{u}\right) / N\right)} \\
=N^{2} \sum_{l=m}^{n} \frac{\pi^{2}\left(l+x T_{u}\right)^{2} / N^{2}}{\sin ^{2}\left(\pi\left(l+x T_{u}\right) / N\right)} \frac{1}{\pi^{2}\left(l+x T_{u}\right)^{2}}
\end{array}
$$

and using the following inequality

$$
1 \leq \frac{1}{\sin c(\theta)}=\frac{\pi \theta}{\sin (\pi \theta)} \leq \frac{\pi}{2}, \quad \theta \in[-0.5,0.5],
$$

we obtain the upper bound for $g(\Delta f, m, n)$ as

$$
\begin{gathered}
\quad g(\Delta f, m, n) \leq \frac{N^{2}}{4} \sum_{l=m}^{n} \frac{1}{(l+\epsilon)^{2}} \\
\leq \frac{N^{2}}{4} \sum_{l=m}^{n}\left\{\frac{1}{(l+\epsilon-0.5)(l+\epsilon+0.5)}\right\} \\
=\frac{N^{2}}{4}\left\{\frac{1}{(m+\epsilon-0.5)}-\frac{1}{(n+\epsilon+0.5)}\right\}
\end{gathered}
$$


where $\epsilon=\Delta f T_{u}$. Substituting the above inequality into (16), we obtain

$$
\operatorname{ICI}_{B}^{i}(k, m, \Delta f) \leq \frac{\pi^{2} \Delta f^{2} T_{u}^{2}}{4}\left(\frac{\left(2 K^{\prime}+1\right)}{\left(\left(K^{\prime}\right)^{2}+K^{\prime}\right) N_{B}}-\frac{4}{K N_{B}}\right) .
$$

The classical Jakes' Doppler spectrum is given by

$$
P_{J}(f)= \begin{cases}\frac{1}{\pi f_{d}} \frac{1}{\sqrt{1-\frac{f^{2}}{f_{d}^{2}}}}, & |f| \leq f_{d} \\ 0, & \text { otherwise }\end{cases}
$$

which can be viewed as the probability density function of Doppler frequency, $P_{J}(f)$. Then the average ICI power is given by

$$
\begin{gathered}
{\overline{\mathrm{ICI}_{B}}}_{B}^{i}(k, m)=\int_{-f_{d}}^{f_{d}} P_{J}(f) \operatorname{ICI}_{B}^{i}(k, m, f) d f \\
\leq \frac{\pi f_{d}^{2} T_{u}^{2}}{4}\left(\frac{\left(2 K^{\prime}+1\right)}{\left(\left(K^{\prime}\right)^{2}+K^{\prime}\right) N_{B}}-\frac{4}{K N_{B}}\right) \times \int_{-1}^{+1} \frac{x^{2}}{\sqrt{1-x^{2}}} d x \\
=\frac{\pi^{2} f_{d}^{2} T_{u}^{2}}{8}\left(\frac{\left(2 K^{\prime}+1\right)}{\left(\left(K^{\prime}\right)^{2}+K^{\prime}\right) N_{B}}-\frac{4}{K N_{B}}\right)
\end{gathered}
$$

which is also attained by combining (10) in [6], (19)-(21), and (16). In Section IV, we will further verify the validity of (24) by simulation. We design $K^{\prime}$ such that the above residual ICI bound is less than a predefined threshold $\gamma$, i.e.,

$$
\frac{\pi^{2} f_{d}^{2} T_{u}^{2}}{8}\left(\frac{\left(2 K^{\prime}+1\right)}{\left(\left(K^{\prime}\right)^{2}+K^{\prime}\right) N_{B}}-\frac{4}{K N_{B}}\right) \leq \gamma
$$

which gives

$$
K^{\prime} \geq-(N-2 \beta K) /(2 N)+\sqrt{N^{2}+4 \beta^{2} K^{2}} /(2 N)
$$

where $\beta=\left(\pi^{2} T_{u}^{2} f_{d}^{2} N\right) /\left(8 \gamma N+4 \pi^{2} T_{u}^{2} f_{d}^{2}\right)$. For example, under the condition of $K=4, N=64, T_{u} f_{d}=0.2$ and $\gamma=-25 \mathrm{~dB}$, we obtain $K^{\prime} \geq 1$ and hence $K^{\prime}=1$ can be used in this case. A smaller residual ICI will require a larger $K^{\prime}$. Actually, $\gamma$ should be inversely proportional to the real-time SNR in channels. For example, it can be defined as $\left(\mathrm{SNR}_{\mathrm{r}}+5\right) \mathrm{dB}$ in order to reduce the residual ICI effect where $\mathrm{SNR}_{\mathrm{r}}$ is the real-time SNR in channels. Below, we further discuss the influence of $N$, NDS $\left(=f_{d} T_{u}\right)$ and $\gamma$ on the choice of $K$ and $K^{\prime}$. Equation (25) is rewritten as

$$
\left(1 /\left(K^{\prime}+1\right)+1 / K^{\prime}\right) K \approx 8 N \gamma /\left(\pi^{2} f_{d}^{2} T_{u}^{2}\right)+4 .
$$

From (28), it is clear that $K^{\prime} / K$ must be reduced when $N$ becomes larger while NDS and $\gamma$ are fixed. Similarly, if NDS is larger and other parameters remain constant, then $K^{\prime} / K$ must be increased.

\section{Simulation Results and Discussions}

Simulations are conducted in Typical Urban (TU) channels with maximum path delay spread $2 \mu \mathrm{s}$. Uncoded system parameters are chosen as follows: the bandwidth of $2 \mathrm{MHz}$, QPSK and 16-QAM with $N=64, L=8$, and subcarrier spacing of $31.25 \mathrm{kHz}$, and 64-QAM with $N=16, L=5$, and subcarrier spacing of $125 \mathrm{kHz}$. SDRIC I, SDRIC II and SDRIC III denote SDRIC with $K=2,4$, and 8, respectively.

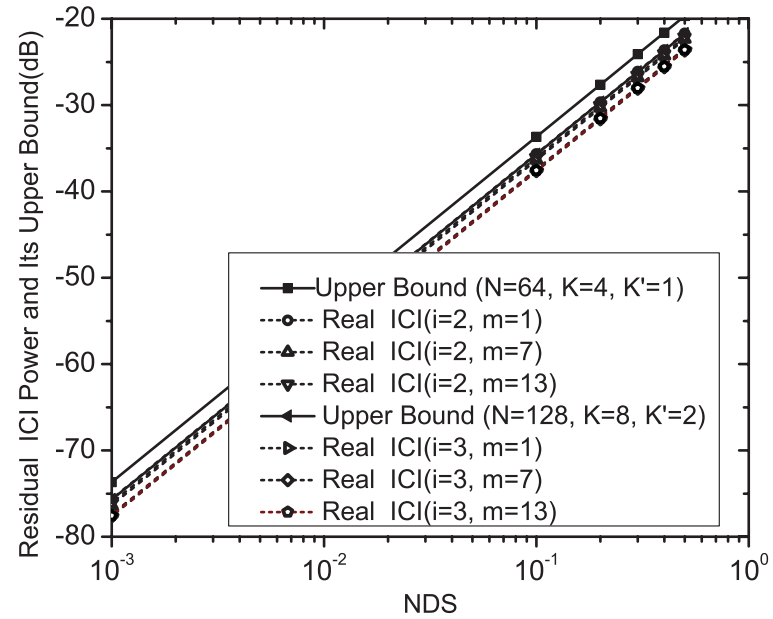

Fig. 1. The residual ICI power versus the normalized Doppler spread (NDS).

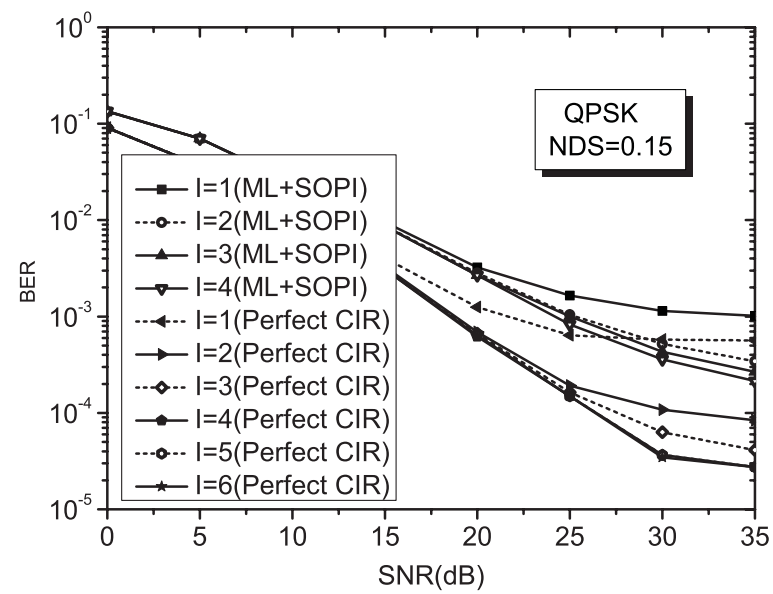

Fig. 2. BER versus SNR for SDRIC at different values of $I$.

\section{A. Simulation Results}

Fig. 1 compares the real residual ICI power in the right side of (18) and its upper bound in (24). From this figure, it is obvious that the curves of real ICI power and its upper bound are parallel and the difference between them is about $2 \sim 3 \mathrm{~dB}$. Thus, the upper bound is a good approximation to the real residual ICI power and can be used as a design metric to calculate $K^{\prime}$.

Fig. 2 shows the BER versus SNR for the proposed SDRIC II with different number of iterations $I$. It is shown that the performance gradually improves as $I$ increases whether ideal CIR or channel estimator ML+SOPI in [4] is used where SOPI represents second-order polynomial interpolation.

Fig. 3 plots the BER versus SNR for SDR, SDRIC, WBDFE with $Q=4$ [8] and PIC with $q=5$ [12] for different values of $K$ when NDS $=0.15$, where $q$ determines the number of taps of the prefilter $(2 q+1$ taps) and the ICI cancellation filter (2q taps) in PIC [12], and $Q$ is the number of subdiagonals and superdiagonals retained in W-BDFE [8]. The performance of SDRIC gradually decreases as $K$ increases. The BER performances of SDRIC I and II are closer to that of SDR and better than PIC and W-BDFE for SNR $>10 \mathrm{~dB}$. The complexity of SDRIC II is far lower than that of SDRIC I. 


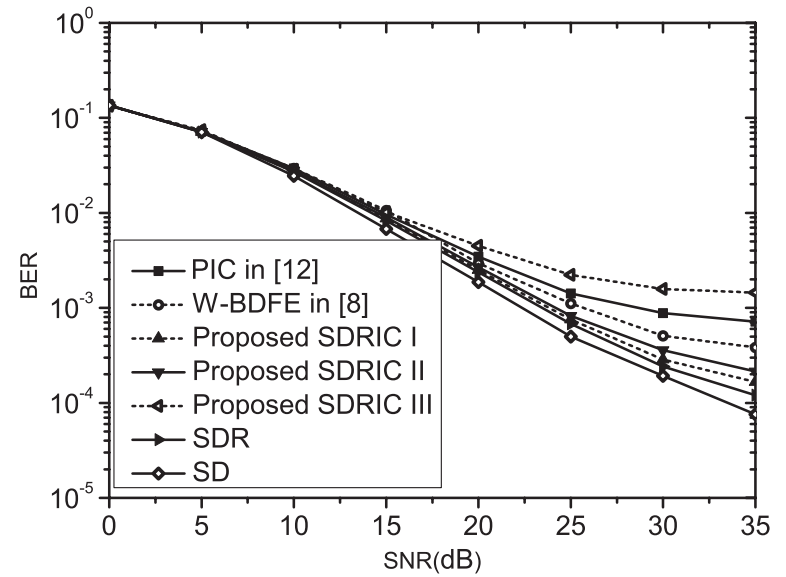

Fig. 3. BER versus SNR for SDR, SDRIC, and PIC (QPSK, NDS=0.15, CIR is estimated by ML+SOPI).

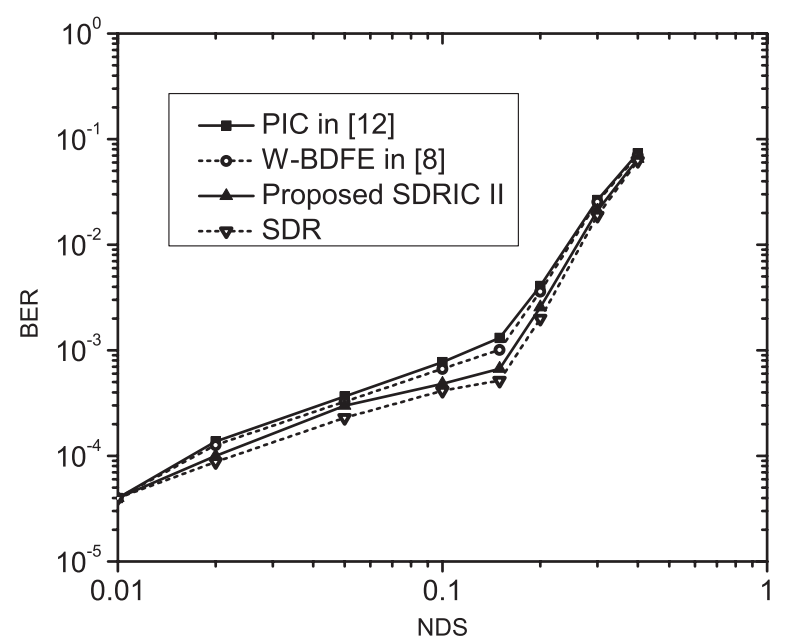

Fig. 4. BER versus NDS for SDR, SDRIC, and PIC (QPSK, SNR=25dB, CIR is estimated by ML+SOPI).

Hence, it is a good choice. The SD in [15] outperforms SDR and SDRIC. Its performance will not be offered below due to its extremely high complexity.

Fig. 4 shows the BER versus NDS for SDR, W-BDFE, PIC and SDRIC II when SNR $=25 \mathrm{~dB}$ and CIR is estimated by ML+SOPI. Their performances become worse as NDS increases. In Fig. 5, the SDR detecting schemes for 16QAM in [21] and for 64-QAM (using (34) with lattice basis reduction) in [23] replace the SDR scheme for QPSK in our SDRIC. The same performance trend is observed as QPSK in Fig. 3. This means our SDRIC can be extended to higher modulation with regular constellation.

\section{B. Complexity Comparisons and Adaptive Detector}

The following simulation considers the computational complexity of SDRIC and other detectors. As shown in Fig. 6, we measure the average numbers of floating point operations (FLOPs) of the following detectors: SDR, PIC [12], W-BDFE with $Q=4$ [8], and SDRIC. From this figure, the complexity of SDRIC II is only one seventh of that of SDR and is slightly more complex than the PIC equalizer. Its performance is better than W-BDFE. Therefore, it is apparent that the

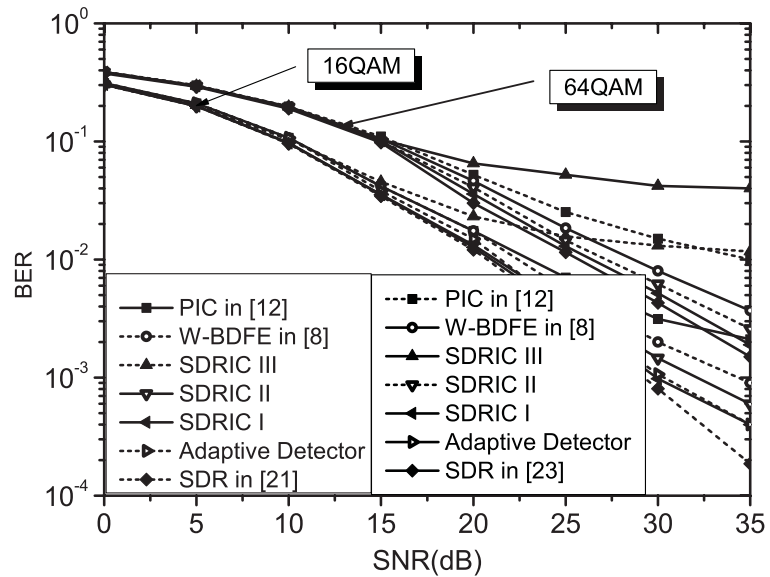

Fig. 5. BER versus NDS for SDR, SDRIC, and PIC (NDS=0.15, ideal CIR).

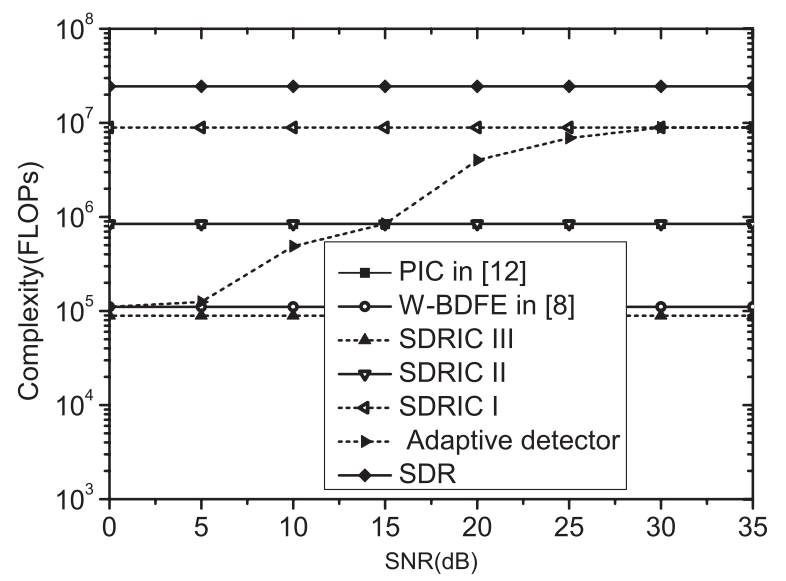

Fig. 6. Complexity comparisons among adaptive detector, SDR, SDRIC, and PIC (16QAM, NDS=0.15, ideal CIR).

proposed SDRIC II strikes a good balance between complexity and performance. However, W-BDFE's low complexity is very attractive. We observe from Fig. 3 and Fig. 5 that i) when $\mathrm{SNR} \leq 10 \mathrm{~dB}$, the performance gap among SDR, SDRIC, W-BDFE, and PIC is approximately zero, ii) when $10 \mathrm{~dB}<\mathrm{SNR} \leq 25 \mathrm{~dB}$, SDRIC II shows lower complexity than and the same performance as SDR and SDRIC I, iii) when SNR $>25 \mathrm{~dB}$, SDRIC I outperforms SDRIC II. Considering their complexity and performance, we propose an adaptive detector as follows: a) The real-time (snap-shot) SNR in channels $\left(\mathrm{SNR}_{\mathrm{r}}\right)$ is computed in advance before detecting where $\mathrm{SNR}_{\mathrm{r}}$ for each OFDM symbol is estimated by the CPbased correlation method (eq.(8) in [27] with the expectation replaced by the sample average); b) If $\mathrm{SNR}_{\mathrm{r}} \leq 10 \mathrm{~dB}, \mathrm{~W}$ BDFE [8] is used ; c) If $10 \mathrm{~dB}<\mathrm{SNR}_{\mathrm{r}} \leq 25 \mathrm{~dB}$, SDRIC II is used; d) If $\mathrm{SNR}_{\mathrm{r}}>25 \mathrm{~dB}$, SDRIC I is adopted. Its performance and complexity are also shown in Fig. 5 and Fig. 6, respectively. From them, it is evident that this detector makes a better balance between complexity and performance compared with other methods.

For a system with a larger number of subcarriers, all considered methods will have higher complexity; but the complexity increase rate is much smaller for the proposed method than the original SDR. For a considered channel environment, the channel delay spread and hence the coherence 
bandwidth are fixed. For the cyclic prefix overhead and the Doppler sensitivity consideration, typically a fixed subcarrier spacing is used for different bandwidths (different numbers of subcarriers) (e.g., see LTE). In our method, as $N_{B}$ is approximately equal to the number of subcarriers within the coherence bandwidth, a larger $N$ will give a larger $K$ but with a fixed $N_{B}$ (approximately). The complexity order of the proposed method depends on $K$ and $N_{B}^{3.5}$, and hence it is linearly proportional to the increase in $N$ since $N_{B}$ is fixed, as opposed to the more-than-cubical increase for the SDR.

\section{CONCLUSiOnS}

In this paper, an SDR detector has been investigated for OFDM systems in DS fading channels. As $N$ increases, SDR's computational amount becomes prohibitive. We have proposed an iterative SDRIC detector to reduce this complexity by a factor of $K^{2.5} I^{-1}$, approximately. Further complexity saving is achieved by considering ICI from $2 K^{\prime}$ closest clusters only. We have derived an upper bound of ICI power from other non-adjacent clusters, and used it as a metric for designing $K^{\prime}$. The simulation results show that the BER performance of the proposed SDRIC is better than that of PIC and slightly worse than that of the original SDR. As the complexity advantage of the proposed SDRIC over the original SDR is quite significant, it provides a good tradeoff between complexity and performance. Finally, an adaptive detector which selects the type of the detector based on the snap-shot SNR estimate is devised and observed to provide a better balance between complexity and performance.

\section{REFERENCES}

[1] M. Speth, S. A. Fechtel, G. Fock, and H. Meyr, "Optimum receiver design for wireless broad-band systems using OFDM," IEEE Trans. Commun., vol. 47, no. 11, pp. 1668-1677, Nov. 1999.

[2] H.-C. Wu, "Analysis and characterization of intercarrier and interblock interferences for wireless mobile OFDM systems," IEEE Trans. Broadcasting, vol. 52, no. 2, pp. 203- 210, June 2006.

[3] W. G. Jeon, K. H. Chang, and Y. S. Cho "An equalization technique for orthogonal frequency-division multiplexing systems in time-variant multipath channels," IEEE Trans. Commun., vol. 47, no. 1, pp. 27-32, Jan. 1999.

[4] F. Shu, Y.-F. Bi, and J.-X. Wang, "Channel estimation and equalization for OFDM wireless system with medium Doppler spread," in Proc. IEEE WiCom, Sep. 2007, vol. 1, pp. 403-407.

[5] Y.-S. Choi, P. J. Voltz, and F. A. Cassara, "On channel estimation and detection for multicarrier signals in fast and selective Rayleigh fading channels," IEEE Trans. Commun., vol. 49, no. 8, pp. 1375-1387, Aug. 2001.

[6] P. Schniter, "Low-complexity equalization of OFDM in doubly selective channels," IEEE Trans. Signal Process., vol. 52, no. 4, pp. 1002-1011, Apr. 2004.

[7] L. Rugini, P. Banelli, and G. Leus, "Simple equalization of time-varying channels for OFDM," IEEE Commun. Lett., vol. 9, no. 7, pp. 619-621, July 2005.
[8] L. Rugini, P. Banelli, and G. Leus, "Low-complexity banded equalizers for OFDM systems in Doppler spread channels," EURASIP J. Applied Signal Process., vol. 2006, pp. 1-13, Jan. 2006.

[9] I. Barhumi, G. Leus, and M. Moonen, "Time-domain and frequencydomain per-tone equalization for OFDM over doubly selective channels," Signal Process., vol. 84, no. 11, pp. 2055-2066, Nov. 2004.

[10] A. Seyedi and G. J. Saulnier, "General ICI self-cancellation scheme for OFDM systems," IEEE Trans. Veh. Technol., vol. 54, no. 4, pp. 198-210, Apr. 2004.

[11] Y. Mostofi and D. C. Cox, "ICI mitigation for pilot-aided OFDM mobile systems," IEEE Trans. Wireless Commun., vol. 5, no. 4, pp. 765-774, Apr. 2005.

[12] W.-S. Hou and B.-S. Chen, "ICI cancellation for OFDM communication systems in time-varying multipath fading channels," IEEE Trans. Wireless Commun., vol. 4, no. 5, pp. 2100-2110, May 2005.

[13] A. Molisch, M. Toeltsch, and S. Vermani, "Iterative methods for cancellation of intercarrier interference in OFDM systems," IEEE Trans. Veh. Technol., vol. 56, no. 4, pp. 2158-2167, Apr. 2007.

[14] E. Viterbo and J. Boutros, "A universal lattice code decoder for fading channels," IEEE Trans. Inf. Theory, vol. 45, no. 7, pp. 1639-1642, July 1999.

[15] M. O. Damen, M. O. El Gamal, and G. Caire, "On maximum-likelihood detection and the search for the closest lattice point," IEEE Trans. Inf. Theory, vol. 49, no. 10, pp. 2389-2402, Oct. 2003.

[16] B. Hassibi and H. Vikalo, "The expected complexity of sphere decoding part I: theory," IEEE Trans. Signal Process., vol. 53, no. 8, pp. 28062818, Aug. 2005.

[17] J. Jalden and B. Ottersten, "On the complexity of sphere decoding in digital communications," IEEE Trans. Signal Process., vol. 53, no. 4, pp. 1474-1484, Apr. 2005.

[18] P. Tan and L. Rasmussen, "The application of semidefinite programming for detection in CDMA," IEEE J. Sel. Areas Commun., vol. 19, no. 8, pp. 1442-1449, Aug. 2001.

[19] W. K. Ma, T. N. Davidson, K. Wong, Z.-Q. Luo, and P.-C. Ching, "Quasi-maximum-likelihood multiuser detection using semi-definite relaxation with application to synchronous CDMA," IEEE Trans. Signal Process., vol. 50, no. 4, pp. 912-922, Apr. 2002.

[20] W. K. Ma, P. C. Ching, and Z. Ding, "Semidefinite relaxation based multiuser detection for M-ary PSK multiuser systems," IEEE Trans. Signal Process., vol. 52, no. 10, pp. 2862-2872, Oct. 2004.

[21] A. Wiesel, Y. Eldar, and S. Shamai, "Semidefinite relaxation for detection of 16-QAM signaling in MIMO channels," IEEE Signal Process. Lett., vol. 12, no. 9, pp. 653-656, Sep. 2005.

[22] N. D. Sidiropoulos and Z. Q. Luo, "A semidefinite relaxation approach to MIMO detection for high-order QAM constellations," IEEE Signal Process. Lett., vol. 13, no. 9, pp. 525-528, Sep. 2006.

[23] A. Mobasher, M. Taherzadeh, R. Sotirov, and A. K. Khandani, "A near maximum likelihood decoding algorithm for MIMO systems based on semi-definite programming," IEEE Trans. Inf. Theory, vol. 53, no. 11, pp. 3869-3886, Nov. 2007.

[24] Y. Yang, C. Zhao, P. Zhou, and W. Xu, "MIMO detection of 16QAM signaling based on semidefinte relaxtion," IEEE Signal Process. Lett., vol. 14, no. 11, pp. 797-801, Nov. 2007.

[25] J. Jalden and B. Ottersten, "The diversity order of the semidefinite relaxation detector," IEEE Trans. Inf. Theory, vol. 54, no. 4, pp. 14061422, Apr. 2008.

[26] P. Moose, "A technique for orthogonal frequency division multiplexing frequency offset correction," IEEE Trans. Commun., vol. 42, no. 10, pp. 2908-2914, Oct. 1994.

[27] J. J. van de Beek, M. Sandell, and P. O. Borjesson, "ML estimation of time and frequency in OFDM systems," IEEE Trans. Signal Process., vol. 45, no. 7, pp. 1800-1805, July 1997. 